\title{
Poorer prognosis in Taiwanese female ever smokers with stage IV lung adenocarcinoma who were readministered a tyrosine kinase inhibitor
}

This article was published in the following Dove Press journal:

OncoTargets and Therapy

I5 March 2016

Number of times this article has been viewed

Chih-Jen Yang, ${ }^{1-4}$ Ming-Ju Tsai, ${ }^{2}$ Jen-Yu Hung, ${ }^{2,4}$ Ying-Ming Tsai, ${ }^{1-3}$ Jui-Ying Lee, ${ }^{5}$ ShahHwa Chou, ${ }^{5,6}$ Ta-Chih Liu, ${ }^{7,8}$ Mei-Chiou Shen, ${ }^{9}$ Ming-Shyan Huang, ${ }^{2,4,10}$ Inn-Wen Chong ${ }^{2,6}$

'Graduate Institute of Medicine, College of Medicine, Kaohsiung Medical University, ${ }^{2}$ Division of Pulmonary and Critical Care Medicine, Department of Internal Medicine, Kaohsiung Medical University Hospital, Kaohsiung Medical University, ${ }^{3}$ Department of Internal Medicine, Kaohsiung Municipal Ta-Tung Hospital, Kaohsiung Medical University Hospital, Kaohsiung Medical University, ${ }^{4}$ Department of Internal Medicine, School of Medicine, College of Medicine, Kaohsiung Medical University, ${ }^{5}$ Division of Chest Surgery, Department of Surgery, Kaohsiung Medical University Hospital, Kaohsiung Medical University, ${ }^{6}$ Department of Respiratory Care, College of Medicine, Kaohsiung Medical University, ${ }^{7}$ Division of Hematology and Oncology, Department of Internal Medicine, Kaohsiung Medical University Hospital, Kaohsiung Medical University, ${ }^{8}$ Institute of Clinical Medicine, College of Medicine, Kaohsiung Medical University, ${ }^{9}$ Department of Pharmacy, Kaohsiung Medical University Hospital, ${ }^{10}$ Division of Geriatric Medicine, Department of Internal Medicine. Kaohsiung Medical University Hospital, Kaohsiung Medical University, Kaohsiung, Taiwan

Correspondence: Inn-Wen Chong; Ming-Shyang Huang

Division of Pulmonary and Critical Care Medicine, Department of Internal Medicine, Kaohsiung Medical University Hospital, Number 100, Tz-You Ist Road, 807 Kaohsiung, Taiwan

Tel +886 73 I2 II 0 I ext 565।

Fax +8867316 I210

Email chong@cc.kmu.edu.tw; shyang@cc.kmu.edu.tw
Background: Readministering a second epidermal growth factor receptor ( $E G F R$ ) tyrosine kinase inhibitor (TKI) in patients with lung adenocarcinoma with acquired resistance to an initial EGFR TKI is a common treatment strategy. However, the prognostic factors for the second EGFR TKI are still uncertain.

Patients and methods: In this retrospective study, we enrolled patients with stage IV lung adenocarcinoma diagnosed between June 2009 and October 2013 at two university-affiliated hospitals in Taiwan. Basic characteristics including age, sex, smoking status, performance status, EGFR mutation status, tumor response, and progression-free survival (PFS) of the second EGFR TKI (PFS2) were recorded.

Results: A total of 72 patients with stage IV adenocarcinoma with susceptible EGFR gene mutations who had been treated with a second EGFR TKI were enrolled. Survival analysis using the Kaplan-Meier method and log-rank test showed a significant difference in PFS2 when classifying the patients according to smoking history and sex $(P=0.0179)$. When stratifying the patients by sex, a significant difference was found in PFS2 between ever smokers and never smokers in the female ( 1.87 vs 4.87 months, $P=0.0081)$ but not in the male ( 2.83 vs 2.9 months, $P=0.9605$ ) patients. A reduced multivariate model developed using the backward variable selection method showed that only ever smoking remained an independent poor prognostic factor for PFS2, and that sex and ever smoking remained independent poor prognostic factors for PFS2 in the female patients (hazard ratio $[\mathrm{HR}]=3.386,95 \%$ confidence interval [CI]: 1.015-11.298, $P=0.0473$ ).

Conclusion: This study is the first to demonstrate that female ever smokers have a poorer PFS if they have acquired resistance to an initial EGFR TKI and receive a second EGFR TKI. Further large-scale studies are urgently needed to elucidate the mechanism.

Keywords: lung cancer, adenocarcinoma, epidermal growth factor receptor tyrosine kinase inhibitor, gefitinib, erlotinib, smoker

\section{Introduction}

Lung cancer is the leading cause of death among patients with malignant tumors worldwide. In 2004, mutations in epidermal growth factor receptor ( $E G F R$ ) that cause oncogene addiction to EGFR were discovered in non-small-cell lung cancer (NSCLC), and these mutations have been found to be strongly associated with sensitivity to $E G F R$ tyrosine kinase inhibitors (TKIs). Several large Phase III trials have shown that EGFR TKIs such as gefitinib and erlotinib can improve the progression-free survival (PFS) of patients with EGFR-mutant NSCLC compared to conventional chemotherapy, although no benefit in overall survival (OS) has been observed. However, the average 
PFS with EGFR TKIs is only $\sim 9-13$ months, and acquired resistance to EGFR TKIs has been shown to develop. ${ }^{1-3}$

In NSCLC patients with EGFR mutations, smoking has been shown to be associated with a shorter PFS than in those who have never smoked, after EGFR TKI treatment in many studies. The lack of an established therapeutic option for NSCLC patients who have progressive disease after failure of EGFR TKI treatment is still a great challenge for physicians. ${ }^{4,5}$ Third-generation EGFR TKIs and combination therapies are currently under investigation, and clinical trials are ongoing. .,7 $^{-7}$ However, these drugs have yet to be approved by the US Food and Drug Administration. Palliative chemotherapy is an option for patients with acquired EGFR TKI resistance. However, the toxicity of antineoplastic agents cannot be tolerated by all patients, and especially the elderly. In 2011, Becker et al demonstrated that retreatment with erlotinib was an option for patients with NSCLC who initially benefited from $E G F R$ TKI treatment and then progressed after standard cytotoxic chemotherapy. ${ }^{8}$ Several small-scale studies and case reports on retreatment with the same or different EGFR TKIs have been published; however, the results have been inconsistent. ${ }^{8-16}$ Therefore, we conducted this retrospective study at two university-affiliated hospitals in Taiwan to investigate the effect of retreatment with different EGFR TKIs in patients with lung adenocarcinoma harboring sensitizing EGFR mutations, and to elucidate the prognostic factors in these patients.

\section{Patients and methods}

\section{Patient identification}

In this retrospective study, patients with stage IV lung adenocarcinoma diagnosed between June 2009 and October 2013 in two university-affiliated hospitals were identified and followed up until September 2014. The diagnosis of lung cancer was confirmed pathologically according to the World Health Organization pathology classification, and tumor staging was determined according to the seventh American Joint Committee on Cancer staging system by a special committee including clinical pulmonologists, medical oncologists, chest surgeons, radiologists, pathologists, and radiation oncologists. Patients were included if they: 1) had adequate tumor specimens for EGFR mutation examinations and 2) were treated with gefitinib and erlotinib in two separate periods.

Baseline clinical characteristics were determined by retrospective chart review, including age at diagnosis, sex, Eastern Cooperative Oncology Group (ECOG) performance status at the beginning of the first TKI treatment and the second TKI treatment, smoking history, and tumor histology. Smoking history was categorized as current smokers or ever smokers, which included ex-smokers (who had quit $\geq 5$ years before the diagnosis), and never smokers ( $<100$ lifetime cigarettes).

Mutations in the EGFR gene were analyzed using an EGFR RGQ kit (Qiagen NV, Venlo, the Netherlands), which utilized amplification refractory mutation specific (ARMS) polymerase chain reactions and Scorpion technology for detection and/or direct sequencing. The detection method was developed and validated by the Division of Molecular Diagnostics, Department of Laboratory Medicine, Kaohsiung Medical University Hospital. An initial treatment response was classified as a complete response (CR), partial response (PR), stable disease (SD), or progressive disease (PD) based on serial imaging studies using the revised Response Evaluation Criteria in Solid Tumors (RECIST 1.1). Disease control was defined as the best tumor response of $\mathrm{CR}, \mathrm{PR}$, or SD that was confirmed and sustained for 8 weeks or longer. The response rate (RR) and disease control rate (DCR) were defined as $\mathrm{CR}+\mathrm{PR}+\mathrm{SD}$. The durations between the initiation of the first TKI and the second TKI to stopping drug treatment due to disease progression were defined as PFS of the first TKI (PFS1) and PFS of the second EGFR TKI (PFS2), respectively. The interval between stopping the first TKI and starting the second TKI was defined as TKIfree interval. OS was defined as the period from starting the second EGFR TKI treatment to the date of death.

\section{Ethics statements}

The study protocol was approved by the Kaohsiung Medical University Hospital Institutional Review Board (KMUHIRB-20120278, KMUHIRB-E(II)-20150162). The Institutional Review Board agreed with waiving the need for written informed consent from the participants due to the retrospective nature of this study.

\section{Statistical analysis}

Age, sex, smoking history, EGFR gene mutation site (exon 19 or others), performance status when starting TKI treatment, and the initial treatment response to the TKI treatment were summarized and compared between ever smokers and never smokers. Categorical variables and continuous variables were compared using the $\chi^{2}$ test (or Fisher's exact test as applicable) and Student's $t$-test, respectively.

Survival times were estimated using the Kaplan-Meier method, with differences between the groups compared using the log-rank test. Cox proportional hazards regression analysis was used to identify the effect of different clinical features on PFS2. After univariate analysis, all variables 
were included to obtain an optimal multivariate model to analyze the independent effect of different variables. We also used the backward variable selection method, keeping only variables with a $P$-value less than 0.1 , to develop a reduced multivariate model. We also performed stratified analyses in both male and female patients. All statistical analyses were performed using SAS software (version 9.3 for Windows; SAS Institute Inc., Cary, NC, USA). Statistical significance was set at a two-sided $P$-value less than 0.05 .

\section{Results}

\section{Patient characteristics}

A total of 72 patients with stage IV adenocarcinoma with susceptible EGFR gene mutations who had been treated with a second $E G F R$ TKI were enrolled. The clinical characteristics of the study population, including EGFR mutation status and PFS, are summarized in Table 1 . There were $55(76 \%)$ never smokers and 17 (24\%) ever smokers. The age was similar between the never smokers $(62.0 \pm 11.8$ years $)$ and ever smokers (60.0 \pm 10.1 years), whereas significantly more male patients were ever smokers. Most (96\%) patients had received gefitinib as the first EGFR TKI and erlotinib as the second EGFR TKI, and only three (4\%) patients had received erlotinib as the first EGFR TKI and gefitinib as the second $E G F R$ TKI. The EGFR mutation status, initial treatment response to the first EGFR TKI, performance status when starting the first and the second EGFR TKI, and TKI-free interval were similar between the never smokers and ever smokers, and no significant difference in PFS1 was noted between the never smokers and ever smokers $(P=0.2027)$.

\section{PFS}

Survival analysis using the Kaplan-Meier method and log-rank test showed a significant difference in PFS2 when

Table I Characteristics of the study population

\begin{tabular}{|c|c|c|c|c|}
\hline Variables & All patients & Never smokers & Ever smokers & $P$-value \\
\hline Patients, n (\%) & $72(100 \%)$ & $55(76 \%)$ & $17(24 \%)$ & \\
\hline Age (years), mean $\pm S D$ & $61.5 \pm 11.4$ & $62.0 \pm 11.8$ & $60.0 \pm 10.1$ & 0.5389 \\
\hline \multicolumn{5}{|l|}{ Age, n (\%) } \\
\hline$<65$ years & $46(64 \%)$ & $34(62 \%)$ & 12 (7|\%) & \multirow[t]{2}{*}{0.5106} \\
\hline$\geq 65$ years & $26(36 \%)$ & $21(38 \%)$ & $5(29 \%)$ & \\
\hline \multicolumn{5}{|l|}{ Sex, n (\%) } \\
\hline Female & $47(65 \%)$ & $43(78 \%)$ & $4(24 \%)$ & \multirow[t]{2}{*}{$<0.0001$} \\
\hline Male & $25(35 \%)$ & $12(22 \%)$ & $13(76 \%)$ & \\
\hline \multicolumn{5}{|l|}{ EGFR gene mutation site, $\mathrm{n}(\%)$} \\
\hline Exon 19 & $39(54 \%)$ & 27 (49\%) & 12 (7I\%) & \multirow[t]{5}{*}{0.1200} \\
\hline Others & $33(46 \%)$ & $28(5 \mid \%)$ & $5(29 \%)$ & \\
\hline Exon 18 & I & 0 & I & \\
\hline Exon 21 & 31 & 27 & 4 & \\
\hline Exon $21+$ exon 20 & I & I & 0 & \\
\hline \multicolumn{5}{|c|}{ Performance status while starting the first TKI, n (\%) } \\
\hline ECOG score $\leq 1$ & $61(85 \%)$ & $46(84 \%)$ & $15(88 \%)$ & \multirow[t]{2}{*}{1.0000} \\
\hline ECOG score $\geq 2$ & II (I5\%) & $9(16 \%)$ & $2(12 \%)$ & \\
\hline \multicolumn{5}{|c|}{ Initial treatment response to the first TKI, n (\%) } \\
\hline Partial response & $50(69 \%)$ & $39(7 \mid \%)$ & II (65\%) & \multirow[t]{3}{*}{0.6873} \\
\hline Stable disease & $16(22 \%)$ & II (20\%) & $5(29 \%)$ & \\
\hline Progressive disease & $6(8 \%)$ & $5(9 \%)$ & I (6\%) & \\
\hline PFSI (months), median (IQR) & $10.2(5.6-14.9)$ & $9.3(4.4-14.7)$ & $11.9(8-15.2)$ & 0.2027 \\
\hline TKI holiday (months), median (IQR) & $4.9(0.6-8.6)$ & $4.6(0.7-8.9)$ & $6.5(0.5-7.9)$ & 0.7153 \\
\hline \multicolumn{5}{|c|}{ Performance status while starting the second TKI, n (\%) } \\
\hline ECOG score $\leq 1$ & $45(63 \%)$ & $33(60 \%)$ & 12 (7I\%) & \multirow[t]{2}{*}{0.4306} \\
\hline ECOG score $\geq 2$ & $27(38 \%)$ & $22(40 \%)$ & $5(29 \%)$ & \\
\hline PFS2 (months), median (IQR) & $3(1.9-5.4)$ & $3.2(2.1-6.4)$ & $2.3(1.4-3.5)$ & 0.2018 \\
\hline \multicolumn{5}{|c|}{ Treatment response to the second TKI, $\mathrm{n}(\%)$} \\
\hline Partial response & $3(4 \%)$ & $3(5 \%)$ & $0(0 \%)$ & \multirow[t]{3}{*}{0.2968} \\
\hline Stable disease & $33(46 \%)$ & $27(49 \%)$ & $6(35 \%)$ & \\
\hline Progressive disease & $36(50 \%)$ & 25 (45\%) & II (65\%) & \\
\hline
\end{tabular}

Notes: The clinical characteristics of the study population, including EGFR mutation status and PFS.

Abbreviations: EGFR, epidermal growth factor receptor; TKI, tyrosine kinase inhibitor; PFSI, progression-free survival of the first TKI; PFS2, progression-free survival of the second EGFR TKI; ECOG, Eastern Cooperative Oncology Group; IQR, interquartile range; SD, standard deviation. 
classifying the patients according to smoking history and sex $(P=0.0179)$ (Figure 1A). In the whole study population, ever smokers had a trend of a worse PFS with the second TKI than the never smokers (median PFS, 2.3 vs 3.2 months; $P=0.0815$ ) (Figure 1B). When stratifying the patients by sex, a significant difference in PFS2 between ever smokers and never smokers was noted in the female patients (1.87 vs 4.87 months, $P=0.0081$ ) (Figure 1C), but not in the male patients (2.83 vs 2.9 months, $P=0.9605$ ) (Figure 1D).

Similarly, univariate Cox regression analysis identified a trend toward a shorter PFS2 in the ever smokers (hazard ratio $[\mathrm{HR}]=1.657,95 \%$ confidence interval $[\mathrm{CI}]$ :
0.930-2.955, $P=0.0869)$ and patients having a poorer performance status ( $\mathrm{ECOG} \geq 2$ ) when starting the second TKI (HR $=1.621,95 \%$ CI: 0.966-2.720, $P=0.0672)$ (Table 2). In multivariate analysis including only smoking history and sex, ever smoking was a significant poor prognostic factor for PFS2 (HR =4.721, 95\% CI: 1.567-14.224, $P=0.0058$ ) (Table 3). However, a significant interaction between smoking history and sex was noted $(P=0.0223)$, suggesting that the effect of smoking history on PFS2 differed between sexes. In multivariate analysis with the optimal model, ever smoking (HR =4.151, 95\% CI: 1.321-13.048, $P=0.0149)$ and having an ECOG $\geq 2$ when starting the second $E G F R$ TKI
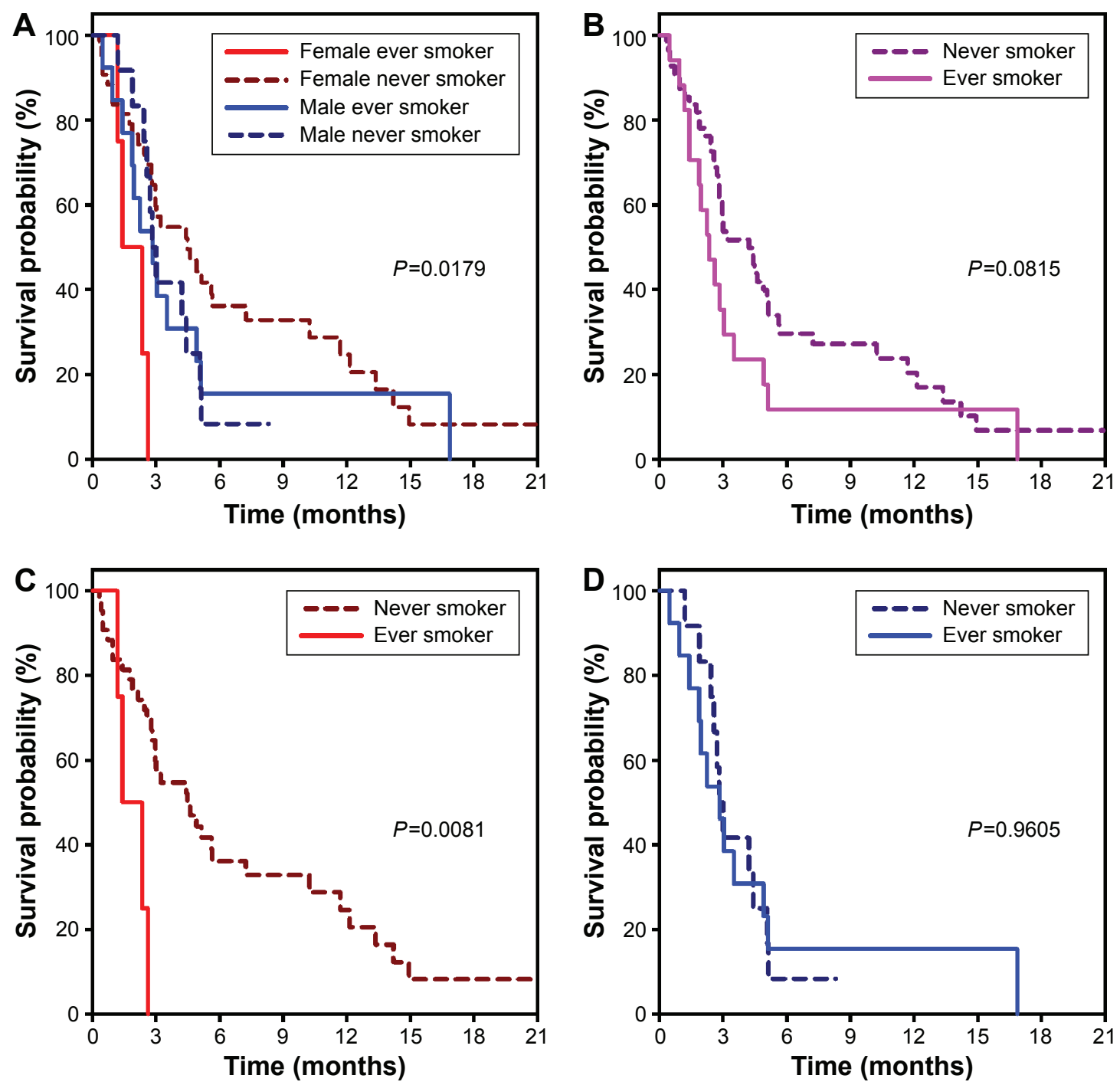

Figure I Survival analysis using the Kaplan-Meier method and log-rank test.

Notes: When classifying the patients according to smoking history and sex, a significant difference in PFS2 was noted ( $P=0.0179)(\mathbf{A})$. In the whole study population, ever smokers had a trend of a worse PFS with the second TKI than the never smokers (median PFS, 2.3 vs 3.2 months, $P=0.0815)(B)$. When stratifying the patients by sex, a significant difference in PFS2 between ever smokers and never smokers was noted in the female patients ( 1.87 vs 4.87 months, $P=0.008 \mathrm{I})$ (C), but not in the male patients (2.83 vs 2.9 months, $P=0.9605)$ (D).

Abbreviations: TKI, tyrosine kinase inhibitor; PFS, progression-free survival; PFS2, progression-free survival of the second EGFR TKI. 
Table 2 Univariate Cox proportional hazards regression analysis to identify the predictive factors for PFS2

\begin{tabular}{lll}
\hline Clinical features & Univariate analysis \\
\cline { 2 - 3 } & HR (95\% CI) & P-value \\
\hline Smoking history (ever vs never smoker) & $1.657(0.930-2.955)$ & 0.0869 \\
Sex (male vs female) & $1.449(0.854-2.458)$ & 0.1687 \\
Age ( $\geq 65$ vs $<65$ years) & $0.909(0.536-1.539)$ & 0.7214 \\
PFSI ( $>10$ vs $\leq 10$ months) & $1.231(0.739-2.049)$ & 0.4252 \\
TKI holiday $(>5$ vs $\leq 5$ months) & $1.185(0.711-1.973)$ & $0.5 I 5 I$ \\
Performance status when starting the second TKI (ECOG $\geq 2$ vs $\leq I)$ & $1.621(0.966-2.720)$ & 0.0672 \\
\hline
\end{tabular}

Notes: Univariate Cox regression analysis identified a trend toward a shorter PFS2 in ever smokers $(\mathrm{HR}=1.657,95 \% \mathrm{Cl}: 0.930-2.955, P=0.0869)$ and in patients having a poorer performance status (ECOG $\geq 2$ ) when starting the second TKI (HR =I.62I, 95\% Cl: 0.966-2.720, $P=0.0672)$.

Abbreviations: TKI, tyrosine kinase inhibitor; PFSI, progression-free survival of the first TKI; PFS2, progression-free survival of the second EGFR TKI; ECOG, Eastern Cooperative Oncology Group; $\mathrm{HR}$, hazard ratio; $\mathrm{Cl}$, confidence interval.

$(\mathrm{HR}=1.810,95 \% \mathrm{CI}: 1.017-3.221, P=0.0436)$ were independent poor prognostic factors for PFS2 (Table 3). Furthermore, the reduced multivariate model developed with the backward variable selection method showed that only ever smoking remained an independent poor prognostic factor for PFS2 (HR =3.814, 95\% CI: 1.247-11.663, $P=0.0189$ ) (Table 3). When stratifying the patients by sex, ever smoking remained an independent poor prognostic factor for PFS2 in the female patients (HR $=3.386,95 \% \mathrm{CI}: 1.015-11.298, P=0.0473)$, but not in the male patients (HR $=0.828,95 \% \mathrm{CI}: 0.323-2.127$, $P=0.6955$ ) (Table 4).

\section{Discussion}

The optimal management for patients with acquired resistance to initial EGFR TKI treatment remains unclear. In this study, we found that female ever smokers had a significantly shorter PFS if they had acquired resistance to EGFR TKIs and then received a second EGFR TKI.
Acquired resistance to EGFR TKIs is common among patients with $E G F R$-mutant lung cancer. Several mechanisms are believed to be responsible for intrinsic and acquired resistance to EGFR TKIs, including secondary EGFR T790M and minor mutations, $M E T$ amplification, activation of the $M E T /$ $H G F$ axis, acquiring an epithelial-to-mesenchymal transition signature, and transformation from NSCLC to small-cell lung cancer. ${ }^{4,5}$ The secondary EGFR mutation, a point mutation in exon 20 (T790M), accounts for approximately half of all cases of acquired resistance to EGFR TKIs. The secondgeneration irreversible $E G F R$ TKI, afatinib, was developed to overcome the T790M point mutation and has shown good efficacy in vitro. However, no clinical benefit was obtained in patients harboring the T790M mutation who received an EGFR TKI in the LUX-Lung series of trials. ${ }^{17}$ Furthermore, several new compounds have been developed to target this point mutation, and clinical trials are currently ongoing. ${ }^{6,7}$ Chemotherapy or giving a second EGFR TKI to NSCLC

Table 3 Multivariate Cox proportional hazards regression analysis to identify the predictive factors for PFS2

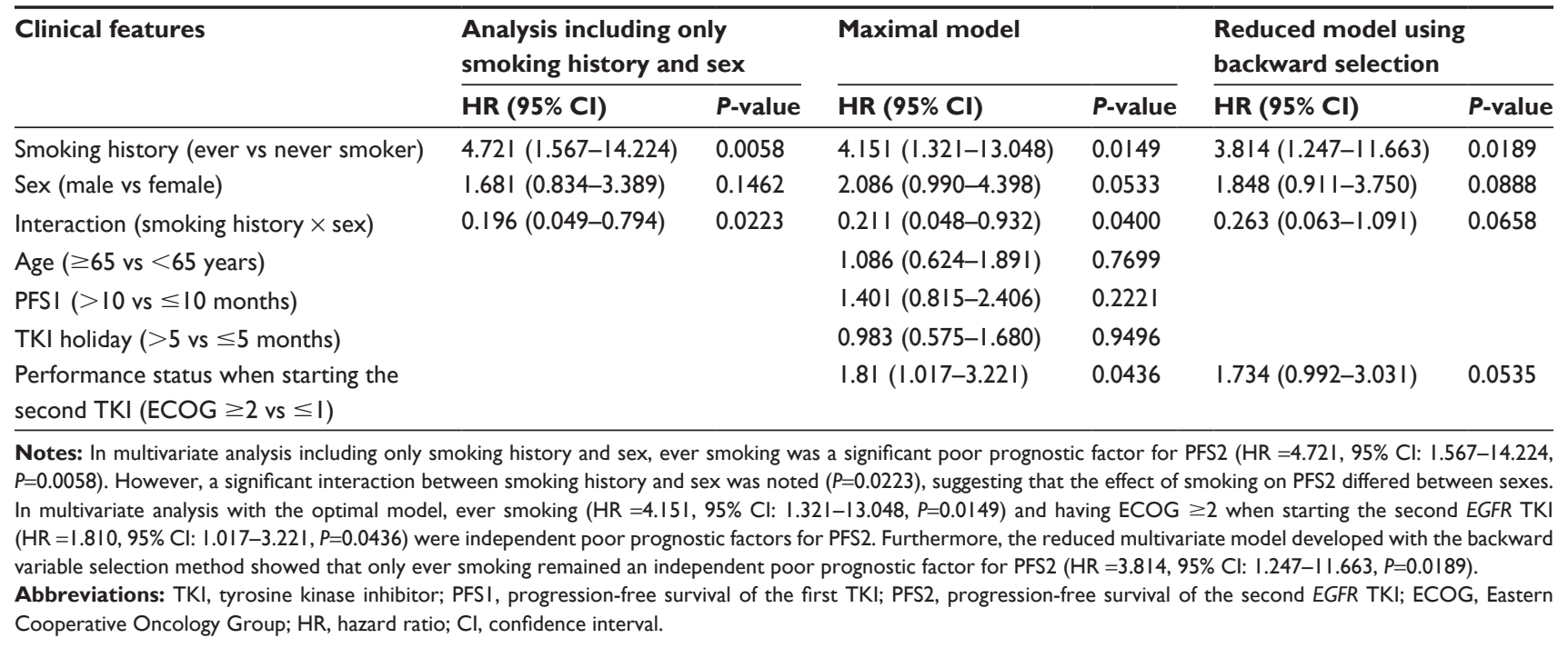


Table 4 Multivariate Cox proportional hazards regression analysis to identify the predictive factors for PFS with a second TKI by sex

\begin{tabular}{|c|c|c|c|c|}
\hline \multirow[t]{2}{*}{ Clinical features } & \multicolumn{2}{|l|}{ Female } & \multicolumn{2}{|l|}{ Male } \\
\hline & HR (95\% Cl) & $P$-value & HR (95\% Cl) & $P$-value \\
\hline Smoking history (ever vs never smoker) & $3.386(1.015-11.298)$ & 0.0473 & $0.828(0.323-2.127)$ & 0.6955 \\
\hline Age ( $\geq 65$ vs $<65$ years) & $0.943(0.465-1.914)$ & $0.87 I I$ & $1.48(0.5$ I $2-4.279)$ & 0.4690 \\
\hline PFS of the first TKI ( $>10$ vs $\leq 10$ months) & I.I84 (0.574-2.44I) & 0.6472 & $2.289(0.786-6.669)$ & 0.1290 \\
\hline TKI holiday ( $>5$ vs $\leq 5$ months) & $0.825(0.396-1.716)$ & 0.6063 & $1.392(0.516-3.754)$ & 0.5139 \\
\hline Performance status when starting the second TKI (ECOG score $\geq 2$ vs $\leq 1)$ & $1.941(0.939-4.010)$ & 0.0733 & $2.615(0.734-9.309)$ & 0.1380 \\
\hline
\end{tabular}

Notes: When stratifying the patients by sex, ever smoking remained an independent poor prognostic factor for PFS2 in the female patients (HR=3.386, 95\% Cl: I.0 I $5-$ II.298, $P=0.0473)$, but not in the male patients ( $H R=0.828,95 \% \mathrm{Cl}: 0.323-2.127, P=0.6955)$.

Abbreviations: $\mathrm{Cl}$, confidence interval; ECOG, Eastern Cooperative Oncology Group; HR, hazard ratio; PFS, progression-free survival; PFS2, progression-free survival of the second EGFR TKI; TKI, tyrosine kinase inhibitor.

patients with disease progression remains controversial. Conventional chemotherapy given after failure of an initial EGFR TKI may also result in a reduction in TKI-resistant clones, leaving TKI-sensitive clones to be further controlled by rechallenge with a second TKI. ${ }^{15}$ Starting a second EGFR TKI is a generally accepted treatment policy in many settings, especially in patients who refuse chemotherapy, the elderly, or in those with a poor performance status after the first $E G F R$ TKI.

The TKI-holiday theory was proposed by Becker et al, who showed a high RR (36\%) and DCR (86\%) in patients who received a second $E G F R$ TKI after a median interval of $\sim 9.5$ (3-36) months. ${ }^{8}$ They concluded that retreatment with erlotinib was an option for patients with NSCLC who initially benefited from $E G F R$ TKI treatment and then experienced recurrence after standard cytotoxic chemotherapy. Sharma et al found that in a human lung cancer cell line (PC9) treated with erlotinib, most cells quickly died; however, a small number of cancer cells persisted. Continued culture of these cells in drug-free media gave rise to drug-sensitive cells (in 9-90 doublings). The authors concluded that altered regulation of chromatin and increased IGF1R activity may be the underlying mechanism for this phenomenon. ${ }^{18}$ Since retreatment with an EGFR TKI was shown to result in a good DCR by Becker et al, ${ }^{8}$ several case reports and case series have been published; however, the results have been inconsistent. According to a review article, the RRs to gefitinib or erlotinib after a drug holiday with the same initial EGFR TKI were $21.3 \%$ for PR, $34.6 \%$ for $\mathrm{SD}$, and $44.1 \%$ for $\mathrm{PD}$, and the DCR for the second EGFR TKI was $55.9 \%$. The median time to progression ranged from 8 to 29 months for TKI therapy, and 1 to 13 months for progression after the initial TKI to retreatment. The strategy of switching between erlotinib and gefitinib (also called replacement therapy) has also been proposed and evaluated. In one study on replacement therapy after failure of the first EGFR TKI, the RRs were $8.2 \%$ for PR, $35.0 \%$ for SD, and $56.8 \%$ for PD. ${ }^{19}$ The results of the current study showed a 4\% PR and 46\% SD rate in all patients. The RR and DCR appeared to be lower in the ever smokers ( $0 \%$ and $35 \%$, respectively) than in the never smokers ( $5 \%$ and $49 \%$, respectively), but without significance.

The clinical characteristics of lung cancer have an impact on molecular presentations such as EGFR mutation status and influence the treatment response in patients with lung cancer. Mitsudomi et al reported that $E G F R$ mutations occur more frequently in Asians, females, nonsmokers, and patients with adenocarcinomas. ${ }^{20}$ Tobacco smoking is a major risk factor for lung cancer in Asia, with reported prevalence rates of smoking among males and females of $65.1 \%$ and $7.1 \%$, respectively. Compared with never smokers, ever smokers were reported to have a higher risk of death from any cause in pooled analyses of all cohorts in 2004, and tobacco smoking has been reported to account for $60.5 \%$ of lung cancer-related deaths in Asian men and $16.7 \%$ in East Asian women. ${ }^{21}$

In patients with lung cancer harboring $E G F R$ mutations, smoking has also been reported to be a poorer prognostic factor when using an EGFR TKI as the first-line treatment. Kim et al reported that smokers with more than a 30 pack-year history had a significantly shorter PFS than never smokers (6.4 vs 11.8 months, $P=0.001$ ) in patients with lung adenocarcinoma, with activating EGFR mutations being regarded as an independent poor prognostic factor. ${ }^{22}$ Chen et al also showed the never smokers had a significantly longer PFS than smokers. ${ }^{23}$ Many hypotheses have been proposed to explain the effect of cigarette smoking on EGFR TKI treatment, such as inducing various critical growth regulatory genes (mostly K-ras and TP53) and inducing aberrant EGFR phosphorylation resulting in a different $E G F R$ conformation and signaling that are resistant to EGFR TKIs. ${ }^{22,23}$ However, precisely who will benefit from treatment with a second $E G F R$ TKI if they have acquired resistance to a first $E G F R$ TKI is still uncertain.

Several prognostic factors of second $E G F R$ TKIs have been proposed. Hata et al suggested that a higher efficacy of erlotinib 
after gefitinib failure can be achieved with proper patient selection criteria, including good performance status, those who benefited from prior gefitinib therapy, and the use of cytotoxic chemotherapies between gefitinib and erlotinib treatment. ${ }^{14}$ In addition, Asami et al indicated that a longer duration of prior gefitinib treatment can predict survival in patients with lung adenocarcinoma who subsequently receive erlotinib. ${ }^{24}$

However, in this study, we found that the prior duration of EGFR TKI treatment, response to the first EGFR TKI, and the TKI-free interval were not significant prognostic factors. After careful statistical analysis, we found that female ever smokers had the shortest PFS of all patients receiving a second EGFR TKI compared with female never smokers (1.87 vs 4.87 months, $P=0.0081$ ) and male ever smokers or male never smokers (2.83 vs 2.9 months). The female patients also had better EGFR TKI response and 1-year PFS rates in a national-wide study in Taiwan. ${ }^{25}$ This suggests that female sex has a positive effect on EGFR TKI treatment for NSCLC. However, smoking may have a greater impact than sex on the effect of EGFR TKI treatment in patients with NSCLC harboring EGFR mutations. Indeed, our results demonstrated that female never smokers had a longer PFS than female ever smokers who received second EGFR TKI treatment. A review of all case reports and case series in PubMed disclosed a good response and longer PFS to a second EGFR TKI treatment; however, almost all female ever smokers have been reported to experience no benefits from a second $E G F R$ TKI, ${ }^{10-13,15,19,26,27}$ which is consistent with our findings.

There are several limitations to this study. First, the sample size is small, although this retrospective analysis may be the largest study to date enrolling patients with stage IV lung adenocarcinoma who initially harbored EGFR mutations and received a second EGFR TKI. Second, almost no patients had a rebiopsy to determine the reason for acquired resistance. Third, almost all of the patients received gefitinib as the first EGFR TKI and erlotinib as the second EGFR TKI. The prescription of gefitinib in patients with lung adenocarcinoma harboring sensitizing EGFR mutations has been permitted by the National Health Insurance (NHI) Bureau in Taiwan since June 2011, but not erlotinib. Therefore, the majority of our enrolled patients were treated with gefitinib as the first EGFR TKI and erlotinib as the second. In addition, retreatment with the same EGFR TKI is not permitted by the NHI Bureau in Taiwan.

\section{Conclusion}

This study indicates that female ever smokers have a significantly shorter PFS when receiving a second EGFR TKI if they initially have lung adenocarcinoma harboring $E G F R$ mutations. Based on these findings, we suggest that in clinical practice, female ever smokers should not be candidates for a second $E G F R$ TKI if they have developed acquired resistance to the first EGFR TKI. Furthermore, large-scale studies and bench research should be conducted to elucidate the detailed mechanism in female smokers using rebiopsy specimens.

\section{Disclosure}

The authors report no conflicts of interest in this work.

\section{References}

1. Mok TS, Wu YL, Thongprasert S, et al. Gefitinib or carboplatinpaclitaxel in pulmonary adenocarcinoma. N Engl J Med. 2009;361(10): 947-957.

2. Maemondo M, Inoue A, Kobayashi K, et al. Gefitinib or chemotherapy for non-small-cell lung cancer with mutated EGFR. $N$ Engl J Med. 2010;362(25):2380-2388

3. Mitsudomi T, Morita S, Yatabe Y, et al. Gefitinib versus cisplatin plus docetaxel in patients with non-small-cell lung cancer harbouring mutations of the epidermal growth factor receptor (WJTOG3405): an open label, randomised phase 3 trial. Lancet Oncol. 2010;11(2):121-128.

4. Gainor JF, Shaw AT. Emerging paradigms in the development of resistance to tyrosine kinase inhibitors in lung cancer. J Clin Oncol. 2013; 31(31):3987-3996.

5. Shien K, Yamamoto H, Soh J, et al. Drug resistance to EGFR tyrosine kinase inhibitors for non-small cell lung cancer. Acta Med Okayama. 2014;68(4):191-200.

6. Walter AO, Sjin RT, Haringsma HJ, et al. Discovery of a mutantselective covalent inhibitor of EGFR that overcomes T790M-mediated resistance in NSCLC. Cancer Discov. 2013;3(12):1404-1415.

7. Cross DA, Ashton SE, Ghiorghiu S, et al. AZD9291, an irreversible EGFR TKI, overcomes T790M-mediated resistance to EGFR inhibitors in lung cancer. Cancer Discov. 2014;4(9):1046-1061.

8. Becker A, Crombag L, Heideman DA, et al. Retreatment with erlotinib: regain of TKI sensitivity following a drug holiday for patients with NSCLC who initially responded to EGFR-TKI treatment. Eur J Cancer. 2011;47(17):2603-2606.

9. Lee DH, Kim SW, Suh C, et al. Phase II study of erlotinib as a salvage treatment for non-small-cell lung cancer patients after failure of gefitinib treatment. Ann Oncol. 2008;19(12):2039-2042.

10. Wong AS, Soong R, Seah SB, et al. Evidence for disease control with erlotinib after gefitinib failure in typical gefitinib-sensitive Asian patients with non-small cell lung cancer. J Thorac Oncol. 2008;3(4): 400-404.

11. Garfield DH. Modern treatment of lung cancer: case 2. Response to erlotinib after failure of gefitinib in a patient with advanced non-smallcell lung carcinoma. J Clin Oncol. 2005;23(30):7738-7740.

12. Chang JW, Chou CL, Huang SF, et al. Erlotinib response of EGFR-mutant gefitinib-resistant non-small-cell lung cancer. Lung Cancer. 2007;58(3): 414-417.

13. Viswanathan A, Pillot G, Govindan R. Lack of response to erlotinib after progression on gefitinib in patients with advanced non-small cell lung cancer. Lung Cancer. 2005;50(3):417-418.

14. Hata A, Katakami N, Yoshioka H, et al. Erlotinib after gefitinib failure in relapsed non-small cell lung cancer: clinical benefit with optimal patient selection. Lung Cancer. 2011;74(2):268-273.

15. Yokouchi H, Yamazaki K, Kinoshita I, et al. Clinical benefit of readministration of gefitinib for initial gefitinib-responders with non-small cell lung cancer. BMC Cancer. 2007;7:51. 
16. Zhao ZR, Li W, Long H. Readministration of EGFR tyrosine kinase inhibitor in non-small cell lung cancer patients after initial failure, what affects its efficacy? Sci Rep. 2014;4:5996.

17. D'Arcangelo M, Hirsch FR. Clinical and comparative utility of afatinib in non-small cell lung cancer. Biologics. 2014;8:183-192.

18. Sharma SV, Bell DW, Settleman J, et al. Epidermal growth factor receptor mutations in lung cancer. Nat Rev Cancer. 2007;7(3):169-181.

19. Song T, Yu W, Wu SX. Subsequent treatment choices for patients with acquired resistance to EGFR-TKIs in non-small cell lung cancer: restore after a drug holiday or switch to another EGFR-TKI? Asian Pac J Cancer Prev. 2014;15(1):205-213.

20. Mitsudomi T, Kosaka T, Yatabe Y. Biological and clinical implications of EGFR mutations in lung cancer. Int $J$ Clin Oncol. 2006;11(3):190-198.

21. Zheng W, McLerran DF, Rolland BA, et al. Burden of total and causespecific mortality related to tobacco smoking among adults aged $\geq 45$ years in Asia: a pooled analysis of 21 cohorts. PLoS Med. 2014; 11(4):e1001631.

22. Kim MH, Kim HR, Cho BC, et al. Impact of cigarette smoking on response to epidermal growth factor receptor (EGFR)-tyrosine kinase inhibitors in lung adenocarcinoma with activating EGFR mutations. Lung Cancer. 2014;84(2):196-202.
23. Chen L, Chen R, Zhu Z, et al. Predictive factors associated with gefitinib response in patients with advanced non-small-cell lung cancer (NSCLC). Chin J Cancer Res. 2014;26(4):466-470.

24. Asami K, Kawahara M, Atagi S, et al. Duration of prior gefitinib treatment predicts survival potential in patients with lung adenocarcinoma receiving subsequent erlotinib. Lung Cancer. 2011;73(2):211-216.

25. Chang $\mathrm{CH}$, Lee $\mathrm{CH}$, Ho $\mathrm{CC}$, et al. Gender-based impact of epidermal growth factor receptor mutation in patients with non-small cell lung cancer and previous tuberculosis. Medicine (Baltimore). 2015; 94(4):e444.

26. Costa DB, Nguyen KS, Cho BC, et al. Effects of erlotinib in EGFR mutated non-small cell lung cancers with resistance to gefitinib. Clin Cancer Res. 2008;14(21):7060-7067.

27. Gridelli C, Maione P, Galetta D, et al. Three cases of long-lasting tumor control with erlotinib after progression with gefitinib in advanced nonsmall cell lung cancer. J Thorac Oncol. 2007;2(8):758-761.
OncoTargets and Therapy

\section{Publish your work in this journal}

OncoTargets and Therapy is an international, peer-reviewed, open access journal focusing on the pathological basis of all cancers, potential targets for therapy and treatment protocols employed to improve the management of cancer patients. The journal also focuses on the impact of management programs and new therapeutic agents and protocols on

\section{Dovepress}

patient perspectives such as quality of life, adherence and satisfaction. The manuscript management system is completely online and includes a very quick and fair peer-review system, which is all easy to use. Visit http://www.dovepress.com/testimonials.php to read real quotes from published authors. 